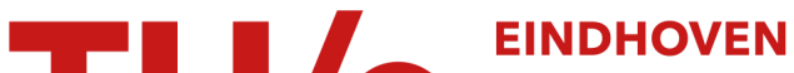 UNIVERSITY OF TECHNOLOGY
}

\section{Enumerating active sites on metal nanoparticles}

\section{Citation for published version (APA):}

van Etten, M. P. C., Zijlstra, B., Hensen, E. J. M., \& Filot, I. A. W. (2021). Enumerating active sites on metal nanoparticles: Understanding the size dependence of cobalt particles for CO dissociation. ACS Catalysis, 11(14), 8484-8492. https://doi.org/10.1021/acscatal.1c00651

DOI:

10.1021/acscatal.1c00651

Document status and date:

Published: 28/06/2021

\section{Document Version:}

Publisher's PDF, also known as Version of Record (includes final page, issue and volume numbers)

\section{Please check the document version of this publication:}

- A submitted manuscript is the version of the article upon submission and before peer-review. There can be important differences between the submitted version and the official published version of record. People interested in the research are advised to contact the author for the final version of the publication, or visit the $\mathrm{DOI}$ to the publisher's website.

- The final author version and the galley proof are versions of the publication after peer review.

- The final published version features the final layout of the paper including the volume, issue and page numbers.

Link to publication

\section{General rights}

Copyright and moral rights for the publications made accessible in the public portal are retained by the authors and/or other copyright owners and it is a condition of accessing publications that users recognise and abide by the legal requirements associated with these rights.

- Users may download and print one copy of any publication from the public portal for the purpose of private study or research.

- You may not further distribute the material or use it for any profit-making activity or commercial gain

- You may freely distribute the URL identifying the publication in the public portal.

If the publication is distributed under the terms of Article 25fa of the Dutch Copyright Act, indicated by the "Taverne" license above, please follow below link for the End User Agreement:

www.tue.nl/taverne

Take down policy

If you believe that this document breaches copyright please contact us at:

openaccess@tue.nl

providing details and we will investigate your claim. 


\title{
Enumerating Active Sites on Metal Nanoparticles: Understanding the Size Dependence of Cobalt Particles for CO Dissociation
}

\author{
Michel P. C. van Etten, Bart Zijlstra, Emiel J. M. Hensen, and Ivo A. W. Filot*
}

Cite This: ACS Catal. 2021, 11, 8484-8492

Read Online

ABSTRACT: Detailed understanding of structure sensitivity, a central theme in heterogeneous catalysis, is important to guide the synthesis of improved catalysts. Progress is hampered by our inability to accurately enumerate specific active sites on ubiquitous metal nanoparticle catalysts. We employ herein atomistic simulations based on a force field trained with quantumchemical data to sample the shape of cobalt particles as a function of their size. Algorithms rooted in pattern recognition are used to identify surface atom arrangements relevant to $\mathrm{CO}$ dissociation, the key step in the FischerTropsch (FT) reaction. The number of step-edge sites that can catalyze $\mathrm{C}-\mathrm{O}$ bond scission with a low barrier strongly increases for larger nanoparticles in

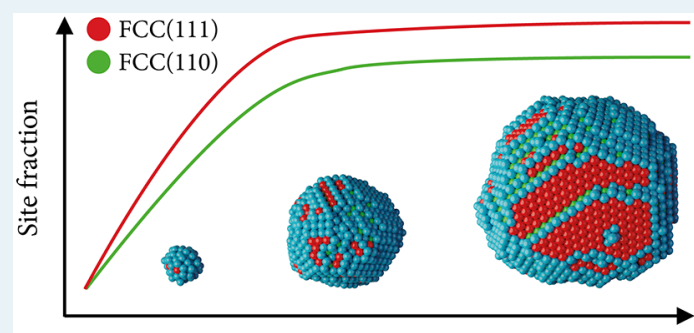

Particle size the range of 1-6 nm. Combined with microkinetics of the FT reaction, we can reproduce experimental FT activity trends. The stabilization of step-edge sites correlates with increasing stability of terrace nanoislands on larger nanoparticles.

KEYWORDS: cobalt, CO hydrogenation, structure sensitivity, nanoparticles, modeling

\section{INTRODUCTION}

Metal nanoparticles constitute an important class of heterogeneous catalysts. The catalytic properties of solids are often significantly changed when the size of nanoparticles is decreased below $10 \mathrm{~nm}$. This phenomenon, commonly referred to as structure sensitivity, is a recurring theme in experimental studies aiming at the optimization of supported metal nanoparticle catalysts. Its importance is clear from the many industrial chemical processes in which the performance of the metal nanoparticle catalysts can be optimized, including steam methane reforming, ${ }^{1-3}$ ammonia synthesis, ${ }^{4-7}$ and Fischer-Tropsch (FT) synthesis. ${ }^{8-13}$ Depending on the reaction, different size-activity trends are observed. Reactions such as steam methane reforming show an increase in surface atom-based catalytic activity with decreasing particle size. In contrast, reactions such as ammonia synthesis and FischerTropsch synthesis display a steep decrease in surface atombased catalytic activity with decreasing particle size. Particle size dependence of catalytic rates is due to a change in the density of crystal planes exposed on the nanoparticle surface as a function of the size. These crystal planes harbor specific atomic arrangements, leading to different intrinsic reactivities toward adsorbed molecules. For example, smaller particles expose more kink and edge sites. The low-coordinated surface atoms facilitate the cleavage of $\sigma$-bonds, an important example being the activation of $\mathrm{C}-\mathrm{H} \sigma$-bonds in $\mathrm{CH}_{4}$. It has been well established by theoretical investigations that the scission of stronger $\pi$-bonds in molecules such as $\mathrm{N}_{2}$ and $\mathrm{CO}$ requires step-edge sites. Larger metal particles are thought to expose more of such step-edge sites, which provides an explanation for the steep increase in catalytic performance with the particle size for ammonia synthesis and Fischer-Tropsch synthesis, requiring the cleavage of the strong intramolecular bonds in, respectively, $\mathrm{N}_{2}$ and $\mathrm{CO} .{ }^{14}$ Honkala et al. showed that $\pi$-bond scission is the rate-determining step in ammonia synthesis and step-edge sites are required to accommodate the dissociation of the triple $\mathrm{N}-\mathrm{N}$ bond. ${ }^{5}$ Although such step-edge sites were observed by transmission electron microscopy (TEM) on Ru nanoparticles, determining their surface density was not possible. Experimentally, determining the exact surface topology as a function of particle size remains a challenge.

Computational modeling has been crucial for understanding structure sensitivity in metal nanoparticle catalysis. In particular, the reactivity of small molecules such as $\mathrm{CO}, \mathrm{NO}$, and $\mathrm{N}_{2}$ on specific surface sites can be studied using state-ofthe-art density functional theory (DFT) using surface slab models that represent the surface facets enclosing nanoparticles. It is equally important to predict the surface atomic arrangements of nanoparticles themselves. Several groups have proposed different procedures for simulating nanoparticles in a realistic manner. ${ }^{15-20}$ Sun et al. simulated ensembles of nanoclusters by first-principles methods to investigate the morphology and catalytic activity. ${ }^{15}$ Cheula et al. combined

Received: February 10, 2021

Revised: June 12, 2021

Published: June 28, 2021 
DFT calculations with Boltzmann statistics to describe ensembles of nanoparticles to obtain different morphologies and their respective active site distribution. ${ }^{16}$ Zhao et al. constructed hcp and fcc $\mathrm{Ru}$ nanoparticles via the Wulff construction according to surface energies obtained by DFT. ${ }^{17}$ Van Helden et al. developed a procedure for building and minimizing particle energies using a lattice model to investigate site distributions in cobalt nanoparticles with an fcc bulk structure as a function of their size. ${ }^{18}$ Agrawal et al. applied molecular dynamics (MD) using an embedded atom model for interatomic interactions to construct cobalt nanoparticles to study bulk behavior at its phase transition temperature. ${ }^{19}$ Rahm et al. presented an algorithm, based on atomistic simulations in a constrained thermodynamic ensemble, to predict changes in equilibrium shapes for nanoparticles in the range of $1-7 \mathrm{~nm}^{20}$

Despite the many valuable insights these studies provide, such modeling efforts have inherent limitations. Although the often used Wulff theorem can be used for dispersion calculations, it suffers from the limitations that edge and corner effects should be neglected so that the outcome is the only representative for particles much larger than the typical sizes at which strong structure sensitivity is observed in experiments. $^{21}$ In Wulff construction and lattice models, it is also assumed that nanoparticles are monocrystalline, while, in practice, metal nanoparticles can be polycrystalline. ${ }^{22}$ To remedy this, we demonstrate a general procedure based on a force field trained from DFT data for optimizing the geometry of nanoparticles without initially imposing any crystal structure.

Modeling structure sensitivity trends for cobalt nanoparticle catalyzed Fischer-Tropsch synthesis requires the use of various simulation techniques covering the relevant length and time scales. In this study, we aim to simulate the surface composition of cobalt nanoparticles as a function of their size and relate the resulting surface composition to the surfacenormalized activity of the Fischer-Tropsch reaction. Our approach comprises four consecutive steps: (i) training a force field (ReaxFF) $)^{23,24}$ based on an extensive set of structural and energetic data from first-principles calculations based on spinpolarized DFT, (ii) optimization of the nanoparticle geometry using simulated annealing-molecular dynamics (MD) simulations, (iii) enumeration of different active sites in terms of surface atom arrangements using a pattern recognition algorithm, and (iv) combining the abundance of such sites with computed reaction rates for $\mathrm{CO}$ consumption to explore structure sensitivity trends. The two most important results are that very small particles show highly disordered surfaces and, with increasing size, the development of facets containing stepedge sites, which are highly reactive toward $\mathrm{C}-\mathrm{O}$ bond scission. The obtained trends are useful for understanding experimental observations and are rationalized in terms of the formation of nanoislands on terraces of larger particles.

\section{COMPUTATIONAL DETAILS}

Density Functional Theory. An extensive training set consisting of structural and energetic data was obtained from quantum-chemical calculations based on spin-polarized density function theory (DFT). The training set contained the equation of state, surface topologies, defect sites, stacking faults, and small clusters. All DFT calculations were performed using the Vienna Ab Initio Simulation package (VASP), using a plane-wave approach in conjunction with the projector- augmented wave (PAW) method for describing the interaction between the nuclei and core electrons. The Perdew-BurkeErnzerhof (PBE) exchange-correlation functional was used to describe the electron-electron interactions. A plane-wave basis set with a kinetic energy cutoff of $400 \mathrm{eV}$ was used for the valance electrons. The $k$-points for the Brillouin zone sampling were dependent on the size of the structures (see Supporting Information). Partial occupancies were determined using a first-order Methfessel-Paxton scheme with a smearing width of $0.2 \mathrm{eV}$. Electronic convergence was set to $10^{-5} \mathrm{eV}$, and geometries were converged to $10^{-4} \mathrm{eV}$ using a conjugategradient algorithm that employs trial and corrector steps to converge both the energy of the structure as well as the forces on the ions. All atoms were allowed to relax. The different surface topologies, defect sites, and stacking faults were modeled in a $3 \times 3$ supercell, consisting of a slab with at least seven layers. To avoid spurious interactions between system images, a vacuum layer of $15 \AA$ was added perpendicular to the surface. The metal slabs were placed at the center of the unit cell, whereas the cobalt clusters were placed at the center of a large unit cell (at least $15 \AA \times 15 \AA \times$ $15 \AA$ ).

We explored reaction paths for $\mathrm{CO}$ dissociation with the climbing image nudged elastic band (cNEB) method. ${ }^{25-27}$ The transition states (TS) were optimized using a quasi-Newton algorithm and confirmed by identifying saddle points using frequency calculations. The Hessian matrices were calculated with the finite displacement technique. The corresponding vibrations were also used to compute zero-point energy (ZPE) corrections and vibrational partition functions for all adsorbed species and transition states.

Reactive Force Field. The ReaxFF reactive force field parameters for cobalt were obtained by fitting ReaxFF parameters with an in-house developed reactive force field fitter $\left(\mathrm{RF}^{3}\right)$ using an extensive training set based on density functional theory data. $\mathrm{RF}^{3}$ uses a genetic algorithm wherein the mutation steps are based on a Markov chain Monte Carlo procedure. Optimization of the force field parameters is done by minimization of the cost function in eq 1

$$
\text { cost function }=\sum_{i=1}^{n} w_{i}\left(x_{i, \mathrm{DFT}}-x_{i, \operatorname{ReaxFF}}\right)^{2}
$$

Herein, $w_{i}$ is a weighing factor, $x_{i, \mathrm{DFT}}$ the binding energy of the structure from the training set, and $x_{i, \text { ReaxFF }}$ the energy of the structure as predicted by the ReaxFF force field.

The optimization of the ReaxFF parameters for a representative chemical system is done in such a way that the reactive force field reproduces a set of reliable energies and structural properties of the various configurations of the system. The training set consists of structural and energetic data obtained from first-principles calculations based on spinpolarized DFT. This set contains (i) the equation of state for different crystalline structures, (ii) crystal surfaces with low Miller indices, (iii) low Miller index crystal surfaces with surface defect sites or stacking faults, and (iv) small clusters in the range of 3-55 atoms.

Molecular Dynamics. Cobalt nanoparticles in a size range of 2-9 $\mathrm{nm}$ were constructed with molecular dynamics using simulated annealing. As the system potential energy is bondorder dependent, the bond orders are updated for every iteration during the $\mathrm{MD}$ run. The MD simulations were performed in the canonical ensemble (NVT) using the velocity 
Verlet algorithm with a time step of $0.25 \mathrm{fs}$. The temperature of the system was controlled using a Nose-Hoover thermostat with a temperature damping constant of $100 \mathrm{fs}$.

For simulated annealing, the atoms were initially positioned in a simple cubic crystal structure to prevent bias toward the final bulk structure. For each particle size, 40 simulations were performed for proper statistical sampling. To differentiate between simulations with the same amount of atoms, the initial velocities of all atoms for each simulation were randomly generated from a Gaussian distribution with a variance $k_{\mathrm{B}} T / m$ using the Mersenne Twister pseudorandom number generator, ${ }^{28}$ with an initial temperature of $1500 \mathrm{~K}$. After a relatively long initialization period at $1500 \mathrm{~K}$, the system is slowly cooled to $300 \mathrm{~K}$ in steps of $100 \mathrm{~K}$.

Surface Atom Arrangement. The surface atom arrangement on the cobalt nanoparticles was acquired with a surface pattern recognition algorithm based on the Common Neighbor Analysis (CNA) method of Reinhart and coworkers. ${ }^{29}$ This method extends the use of coordination numbers by taking into account the connections between the first shell of nearest neighbors and classifying particles into one of a set of reference structures by analysis of the local geometry as defined by a cutoff distance. This cutoff distance is typically around 1.4 times the average distance to the nearest six atoms, corresponding to the first coordination shell of the metal atom. This approach decomposes the overall radial distribution function (RDF) in contributions of different surface structures. To calculate the surface density of a particular active site, all atoms were classified as either surface atoms or bulk atoms. Atoms with a coordination number lower than 12 were herein designated as surface atoms and those with a coordination number of 12 as bulk atoms.

\section{RESULTS AND DISCUSSION}

Molecular Dynamics. To explore cobalt nanoparticle geometry as a function of size, we employed simulated annealing-molecular dynamics (MD) simulations based on a reactive force field. The ReaxFF force field was trained and validated with first-principles DFT data as shown in the Supporting Information (Figure S1, Tables S1-S8). Figure S1 shows an overview of the minimization of the cost function as a function of the number of iterations. Herein, it can be seen that there is a steep decrease in the cost function within the first 10 iterations, a slow decrease until around 200 iterations, and no significant improvement in the fit after 200 iterations. Tables S1-S5 shows that with the exception of some of the small cobalt clusters, the difference between the target energies of the DFT training set and the obtained energies from the ReaxFF force field were all below $0.1 \mathrm{eV}$ per atom. Therefore, the accuracy of our force field is below $0.1 \mathrm{eV}$ per atom. Note that this criterion is an upper limit, while the largest differences between DFT and ReaxFF are observed for bulk systems that are compressed and for small clusters that contain undercoordinated cobalt atoms. An overview of the deduced reactive force field parameters can be found in Tables S6-S8. To verify that the trained force field is accurate enough for simulating small cobalt nanoparticles of 125 and 216 atoms, we benchmarked the resulting nanoparticle formation energy from ReaxFF with the corresponding formation energy obtained from single-point DFT calculations, as shown in Figure S2. The parity plot in Figure S2 shows that the difference in the formation energy for the small cobalt
Table 1. Number of Iterations during Initialization and Per Annealing Step, Average Particle Size and Corresponding Standard Deviation for Each of the Simulated Systems with Constant Number of Cobalt Atoms

\begin{tabular}{|c|c|c|c|c|}
\hline $\begin{array}{l}\text { number } \\
\text { of atoms }\end{array}$ & $\begin{array}{c}\text { number of } \\
\text { iterations during } \\
\text { initialization at } \\
1500 \mathrm{~K}\end{array}$ & $\begin{array}{c}\text { number of } \\
\text { iterations per } \\
\text { annealing step of } \\
100 \mathrm{~K}\end{array}$ & $\begin{array}{c}\text { average } \\
\text { particle } \\
\text { size }(\mathrm{nm})\end{array}$ & $\begin{array}{l}\text { standard } \\
\text { deviation } \\
(\mathrm{nm})\end{array}$ \\
\hline 125 & 200000 & 20000 & 1.4 & 0.005 \\
\hline 216 & 200000 & 20000 & 1.7 & 0.006 \\
\hline 343 & 200000 & 20000 & 2.1 & 0.007 \\
\hline 512 & 200000 & 20000 & 2.4 & 0.008 \\
\hline 729 & 200000 & 20000 & 2.7 & 0.009 \\
\hline 1000 & 200000 & 20000 & 3.0 & 0.008 \\
\hline 1331 & 200000 & 20000 & 3.3 & 0.014 \\
\hline 1728 & 200000 & 20000 & 3.6 & 0.012 \\
\hline 2197 & 200000 & 20000 & 3.9 & 0.016 \\
\hline 2744 & 200000 & 20000 & 4.2 & 0.013 \\
\hline 3375 & 200000 & 20000 & 4.5 & 0.019 \\
\hline 4096 & 400000 & 40000 & 4.8 & 0.015 \\
\hline 4913 & 400000 & 40000 & 5.1 & 0.017 \\
\hline 5832 & 400000 & 40000 & 5.4 & 0.018 \\
\hline 6859 & 400000 & 40000 & 5.7 & 0.017 \\
\hline 8000 & 400000 & 40000 & 6.0 & 0.022 \\
\hline 9261 & 600000 & 60000 & 6.3 & 0.021 \\
\hline 12167 & 600000 & 60000 & 6.9 & 0.017 \\
\hline 15625 & 600000 & 60000 & 7.5 & 0.015 \\
\hline 27000 & 900000 & 90000 & 9.1 & 0.020 \\
\hline
\end{tabular}

nanoclusters is below $0.02 \mathrm{eV} /$ atom, showing good agreement between DFT and ReaxFF.

For the MD optimization of nanoparticle geometry, the number of iterations and simulated annealing trajectories depend on the size of the studied system. An overview of the number of iterations per different particle sizes is shown in Table 1. For each set of simulated systems with a constant amount of atoms, the average particle size and the corresponding standard deviation is also shown in Table 1 . The particle size of each resulting cobalt nanoparticle was obtained by determining the largest distance between two atoms within the system. The average particle size was obtained by statistical averaging of the 40 simulations.

From Table 1, it can be seen that the standard deviation for all simulated particle sizes is sufficiently low, meaning that the particle size distribution is narrow and evenly spread. A representative example of an MD trajectory of a $3 \mathrm{~nm}$ cobalt nanoparticle is shown in Figure 1. After initialization at $1500 \mathrm{~K}$ of the cubic lattice particle, the structure rapidly changes into a more spherical shape with crystal facets appearing as annealing proceeds. A movie corresponding to the simulated annealing trajectory of Figure 1 can be found in the Supporting Information.

Convergence of these MD simulations was evaluated from the radial distribution functions ( $R D F s$ ) of the optimized nanoparticle geometries. In all cases, the RDFs correspond to those expected for a bulk phase composed of fcc and hcp crystalline cobalt, which is consistent with the X-ray diffraction (XRD) patterns of practical cobalt catalysts. ${ }^{21,30}$ The influence of faster cooling on the obtained geometries is exemplified for a $4.5 \mathrm{~nm}$ cobalt nanoparticle in Figure S3 (Supporting Information). Too fast cooling leads to an amorphous bulk structure, while a preference for an fcc bulk structure develops when the annealing trajectories are not long enough. In this 


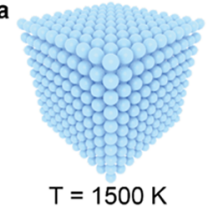

$T=1500 \mathrm{~K}$

$\mathrm{t}=0 \mathrm{ps}$

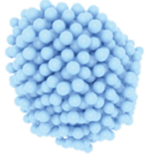

$\mathrm{T}=1000 \mathrm{~K}$

$\mathrm{t}=70 \mathrm{ps}$ b

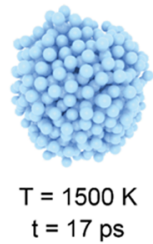

g

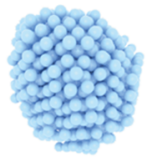

$\mathrm{T}=800 \mathrm{~K}$

$\mathrm{t}=80 \mathrm{ps}$
C

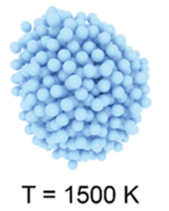

$\mathrm{t}=33 \mathrm{ps}$

h

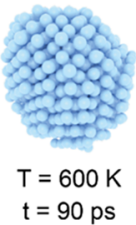

d

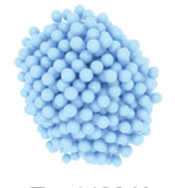

$T=1400 \mathrm{~K}$ $\mathrm{t}=50 \mathrm{ps}$

i

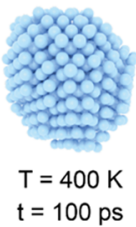

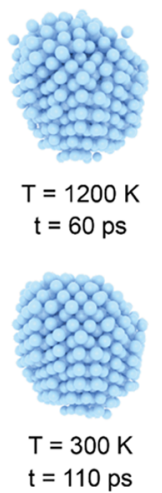

Figure 1. Snapshots of a MD trajectory of a $3 \mathrm{~nm}$ cobalt nanoparticle. Note that for the visualization, the radii of the atoms are slightly decreased to better show the position of the subsurface atoms.

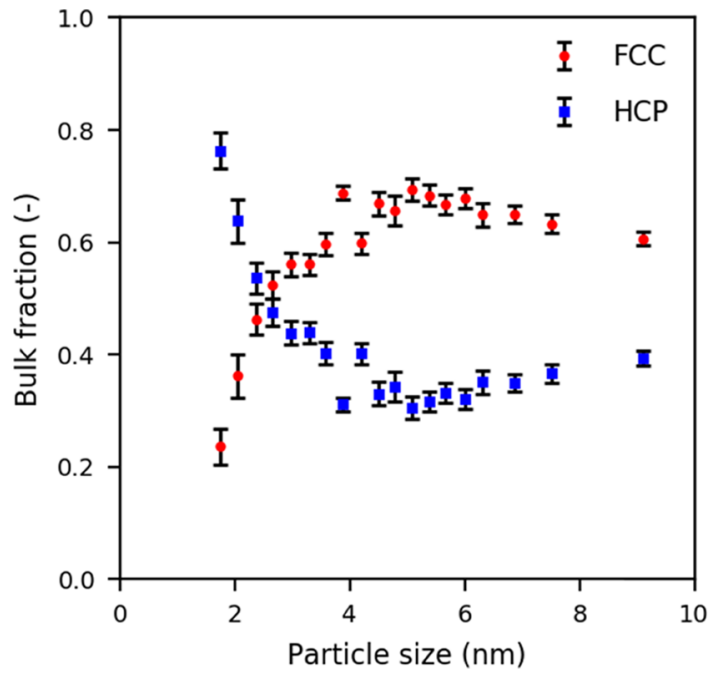

Figure 2. Average fraction of fcc and hcp bulk phases as a function of the cobalt particles size. The error bars show the $95 \%$ confidence interval. way, we optimized the MD procedure and investigated the influence of size on cobalt nanoparticle geometry.

The bulk compositions were analyzed for each of the simulated cobalt nanoparticles by counting all atoms with either an fcc or hcp bulk configuration and dividing them by the total amount of atoms with a bulk configuration. For each of the simulated particle sizes, the average bulk fractions were obtained by statistical averaging. As the accuracy of our force field is similar to the energy difference between the hcp and fcc crystal structures of cobalt $(\sim 0.02 \mathrm{eV} /$ atom $)$, we should not a priori expect to be able to predict preferred bulk structures. Nevertheless, our bulk analysis, as shown in Figure 2, shows that particles larger than $4 \mathrm{~nm}$ mainly have the fcc structure. This prediction is in line with the experimental findings of Kitakami et al. that the fcc bulk phase is more stable than the hcp bulk phase for particles smaller than $20 \mathrm{~nm} .{ }^{31}$ Analyzing the structures of annealed cobalt particles smaller than $4 \mathrm{~nm}$, which are not commonly amenable to XRD analysis in practice, shows however that hcp motives become more dominant than fcc ones. a

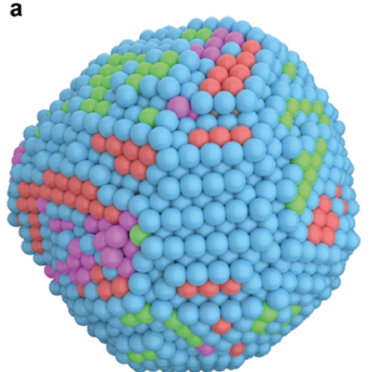

c

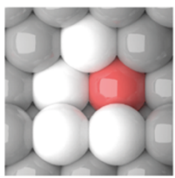

$\operatorname{FCC}(211)$

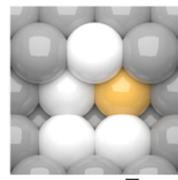

$\operatorname{HCP}(01 \overline{1} 2)$

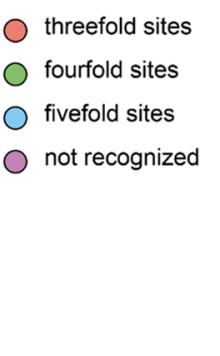

b

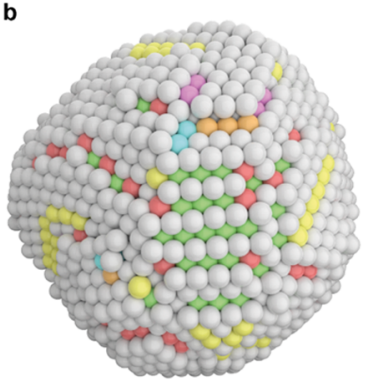

$\operatorname{FCC}(211)$

$\mathrm{HCP}(01 \overline{1} 2)$

$\mathrm{HCP}(01 \overline{1} 1)$

FCC $(110)$

$\mathrm{HCP}(03 \overline{3} 1)$

$\mathrm{HCP}(01 \overline{1} 3)$

other

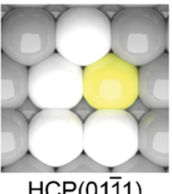

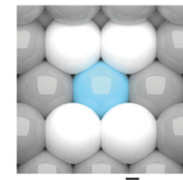

$\operatorname{HCP}(03 \overline{3} 1)$

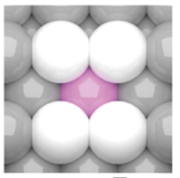

$\mathrm{HCP}(01 \overline{1} 3)$

Figure 3. Surface analysis of an annealed cobalt nanoparticle of $\sim 6 \mathrm{~nm}$ by the CNA algorithm: (a) terrace sites (threefold and fourfold sites) and step-edge sites (fivefold sites), (b) different types of step-edge sites, and (c) local topology of step-edge sites with colored identifier atoms corresponding to panel b. (a) Threefold sites in red, fourfold sites in green, fivefold sites in blue, and atom arrangements not recognized into one of a set of reference structures in pink. (b) step-edge sites with identifiers corresponding to $\operatorname{FCC}(211), \operatorname{HCP}(01 \overline{1} 2), \operatorname{HCP}(01 \overline{1} 1), \operatorname{FCC}(110)$, $\mathrm{HCP}(03 \overline{3} 1)$, and $\mathrm{HCP}(01 \overline{1} 3)$ orientation in red, orange, yellow, green, blue, and pink, respectively, and all other atoms in gray. (c) For each of the step-edge sites $(\operatorname{FCC}(211), \operatorname{HCP}(01 \overline{1} 2), \operatorname{HCP}(01 \overline{1} 1), \operatorname{FCC}(110), \operatorname{HCP}(03 \overline{3} 1)$, and $\operatorname{HCP}(01 \overline{1} 3))$, the identifier atoms are shown in red, orange, yellow, green, blue and pink, respectively. 

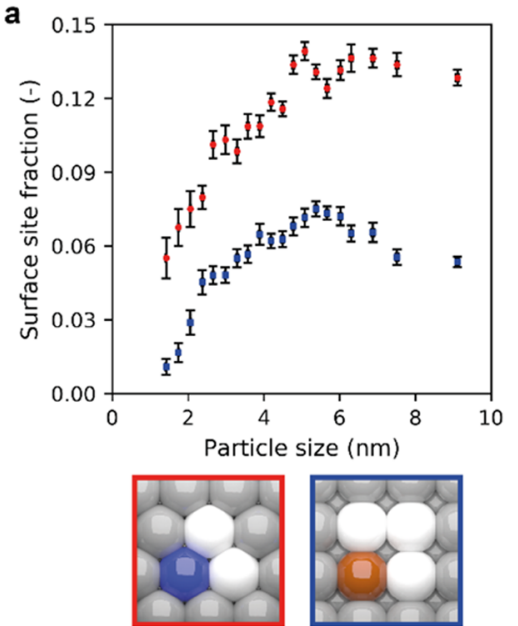

$\mathrm{FCC}(111)$

d

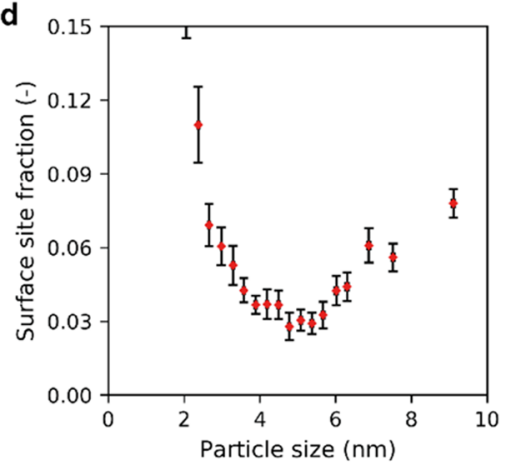

b
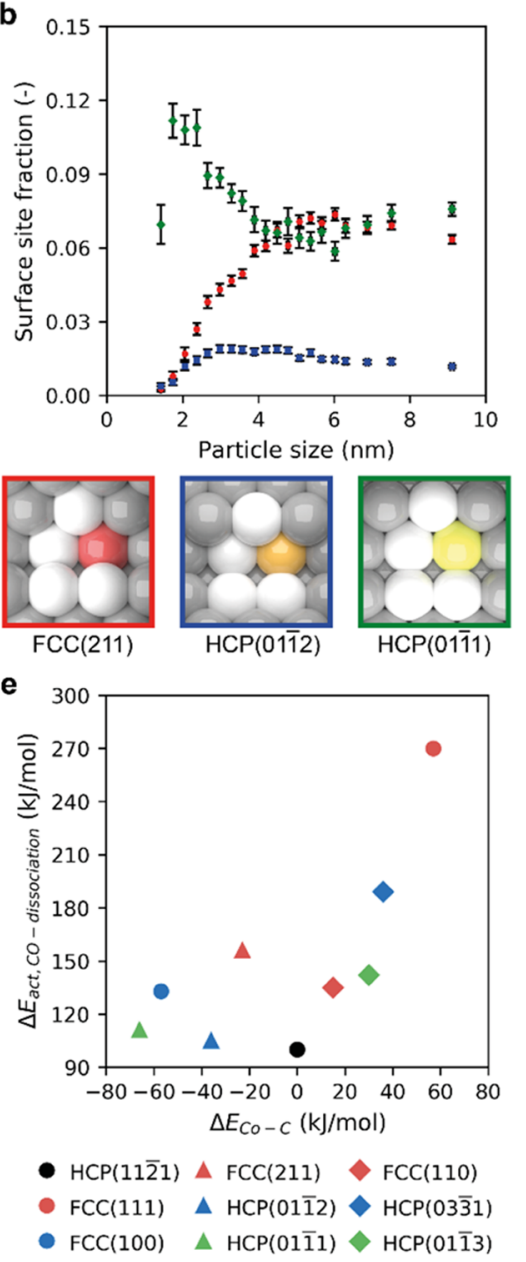

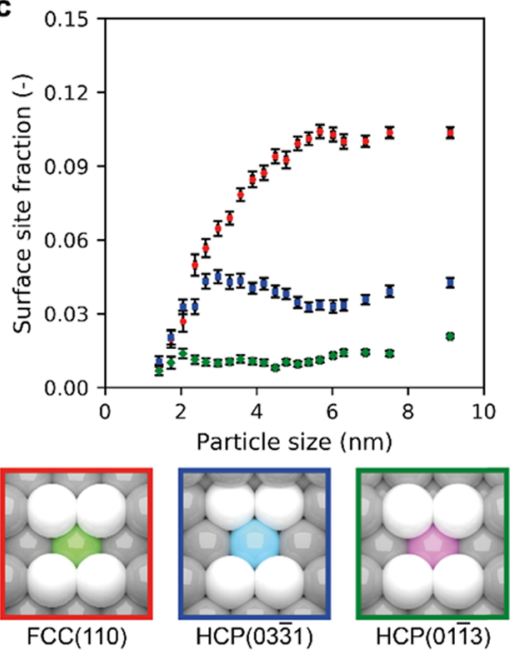

f

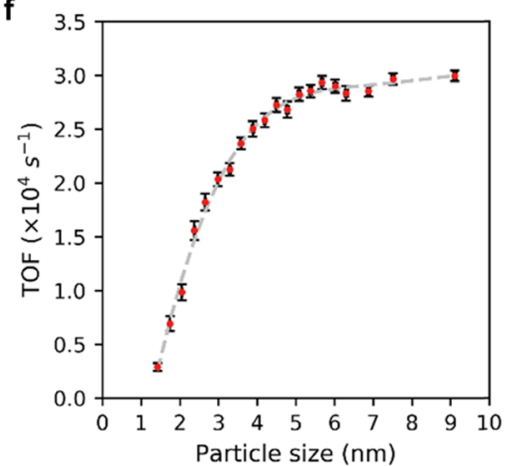

Figure 4. Abundance of terrace sites (a), step-edge sites (b, c), and low-coordinated surface atoms (d) as functions of the particle size, (e) twodimensional (2D) plot of cobalt-carbon interaction strength for each terrace/step-edge site with respect to the $\mathrm{HCP}(11 \overline{2} 1)$-oriented step-edge site and the activation energy for $\mathrm{CO}$ dissociation, and (f) surface atom-based turnover frequency as a function of the nanoparticle size. (a) Terrace sites of orientations (red) FCC(111) and (blue) FCC(100). (b) Step-edge sites of orientations (red) FCC(211), (blue) HCP(0112), and (green) $\mathrm{HCP}(01 \overline{1} 1)$. (c) Step-edge sites with orientations (red) FCC(110), (blue) $\mathrm{HCP}(03 \overline{3} 1)$, and (green) $\mathrm{HCP}(01 \overline{1} 3)$. (d) (red) Low-coordinated surface atoms not recognized by the CNA algorithm. (e) Reference site with $\operatorname{HCP}(11 \overline{2} 1)$ orientation (black dot), terrace sites with orientations (red dot) FCC(111) and (blue dot) FCC(100), step-edge sites with orientations (red triangle) FCC(211), (blue triangle) $\mathrm{HCP}(01 \overline{1} 2)$, (green triangle) $\mathrm{HCP}(01 \overline{1} 1)$, (red diamond) $\mathrm{FCC}(110)$, (blue diamond) $\mathrm{HCP}(03 \overline{3} 1)$, and (green diamond) $\mathrm{HCP}(01 \overline{1} 3)$. (f) Dashed gray line serves as a guide to the eyes. Error bars correspond to the $95 \%$ confidence intervals determined by averaging over 40 simulations of particles of the same number of atoms. Note that $95 \%$ confidence interval for the particle size is less than $0.05 \mathrm{~nm}$ by which the horizontal error bars are indiscernible.

Surface Site Composition. As the reactivity of the surface toward molecular adsorbates usually involves more than one surface metal atom, it is necessary to enumerate specific topological arrangements of surface metal atoms rather than to classify surface metal atoms solely on the basis of their coordination numbers. ${ }^{19,31}$ The common neighbor analysis (CNA) method is used to enumerate such sites. We assembled a database of reference surface topologies, which are given in Table S9. This analysis is limited to the first shell of neighbors of a cobalt atom, which is sufficient to find surface structureactivity correlations with respect to activation of $\mathrm{CO}$. The main assumption is that the surface is static during the ongoing catalytic reaction. To study dynamic aspects of the surface under Fischer-Tropsch conditions, the current ReaxFF force field parameters have to be extended with cobalt/carbon/ oxygen/hydrogen interactions and trained against an extended DFT training set.

Figure 3 shows a cobalt nanoparticle $(\sim 6 \mathrm{~nm})$. In Figure 3a, threefold, fourfold, and fivefold sites are indicated in red, green, and blue, respectively. Step-edge sites are represented by different colors using an identifier atom in Figure $3 \mathrm{~b}$. The six different step-edge sites are shown in Figure 3c. The pink atoms in Figure 3 a represent atoms that were not recognized by the CNA algorithm within the framework of our database of reference structures.

Figure $4 a-d$ shows how the surface composition in terms of topological arrangements (terrace sites, step-edge sites, and low-coordinated atoms) changes with the nanoparticle size. The abundance of terrace and step-edge sites with, respectively, FCC(211) and FCC(110) orientations increases with the size up to $\sim 6 \mathrm{~nm}$ and then levels off. Step-edge sites with $\operatorname{HCP}(01 \overline{1} 2)$ and $\operatorname{HCP}(03 \overline{3} 1)$ orientations are most abundant on $\sim 3 \mathrm{~nm}$ sized particles, while those with the $\operatorname{HCP}(01 \overline{1} 1)$ orientation display a maximum for $\sim 2 \mathrm{~nm}$ particles. $\operatorname{HCP}(01 \overline{1} 3)$-oriented step-edge sites are present in very small amounts over the whole size range. Figure $4 d$ demonstrates that the surfaces of smaller particles contain more atoms that cannot be identified by the CNA algorithm. 

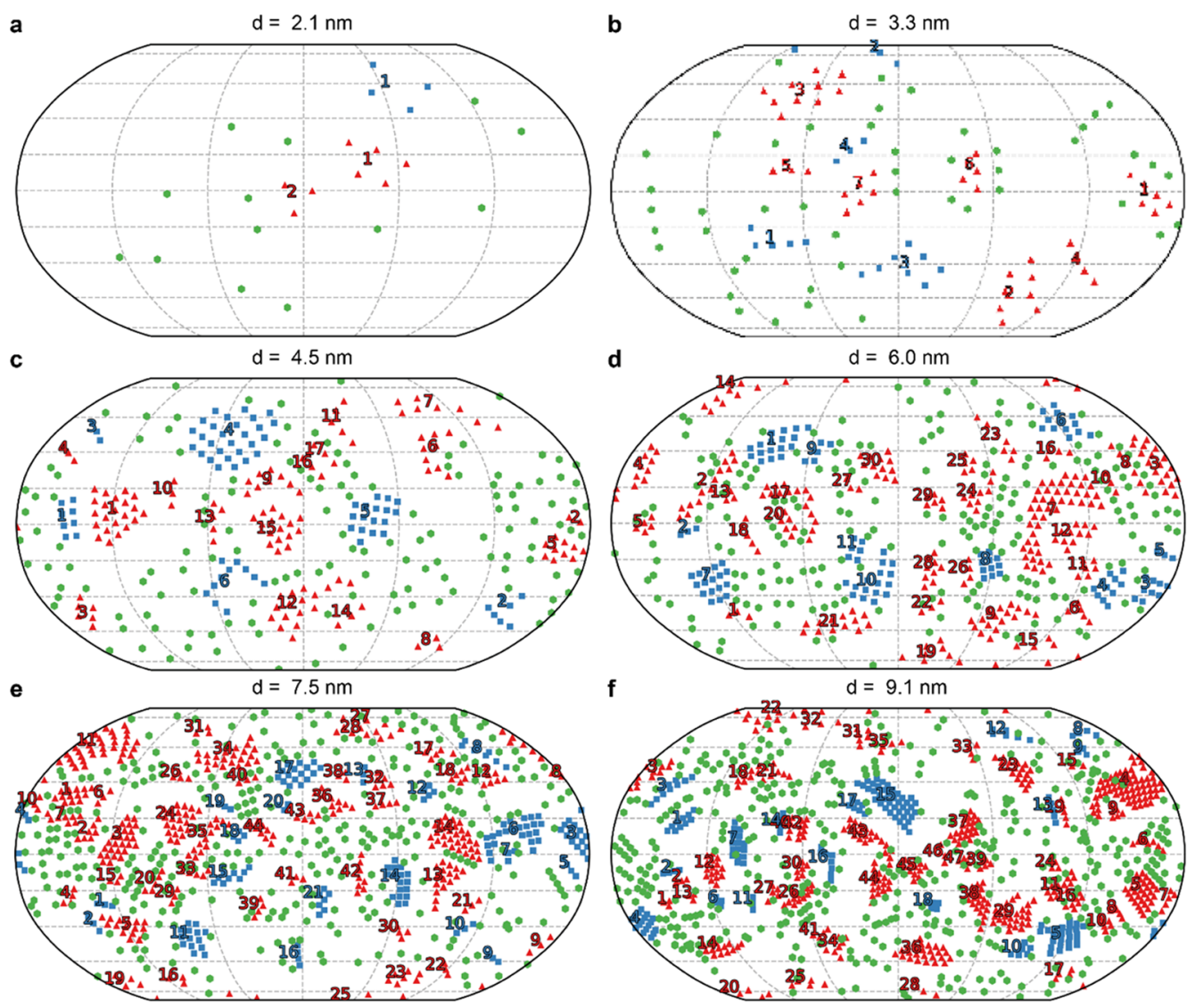

Figure 5. 2-D Robinson projections of differently sized cobalt nanoparticles: (a) $2.1 \mathrm{~nm}$, (b) $3.3 \mathrm{~nm}$, (c) $4.5 \mathrm{~nm},(\mathrm{~d}) 6.0 \mathrm{~nm},(\mathrm{e}) 7.5 \mathrm{~nm}$, and (f) $9.1 \mathrm{~nm}$. Threefold terrace sites in red, fourfold terrace sites in blue and fivefold step-edge sites (FCC $(110))$ in green. An increase in the terrace nanoislands size with increasing particle size can be seen, as well as the step-edge sites are surrounding the terrace nanoislands.

Most of these atoms have coordination numbers 5, 6, or 7, which implies that they are edge, corner, and kink atoms. Such low-coordinated sites are usually assumed to be prominent on very small particles and important to $\mathrm{C}-\mathrm{H}$ bond activation in alkanes. ${ }^{1}$ On the other hand, they cannot dissociate molecules like $\mathrm{CO}$ that are relevant to the FT reaction. The surface contribution of such low-coordinated atoms is less than $10 \%$ on particles larger than $2.5 \mathrm{~nm}$.

Structure Sensitivity. The unusual structure sensitivity of the heterogeneous FT reaction requires a description beyond the usual Langmuir assumption of a uniform surface. Instead, the surface reactivity of the cobalt nanoparticles is described in the Taylorian framework, in which catalysis occurs at uniquely active sites that may be present in very small numbers compared to other less active sites. ${ }^{31}$ The particle-based rate is the sum of rates of a site normalized by its abundance on a particle of a given size. We predict site-based rates for each of the terrace and step-edge sites identified on cobalt nanoparticles using an extensive microkinetic model under the static surface assumption based on DFT-computed reaction energetics for the cobalt $\operatorname{HCP}(11 \overline{2} 1)$ surface. ${ }^{32,33}$ Coverage effects are included in the microkinetic model by means of lateral interactions. We computed $\mathrm{CO}$ dissociation barriers for each of the identified sites with DFT (Figure 4e, Table S10). We found that the $\mathrm{CO}$ dissociation barriers varied only slightly with structural differences of the active sites of the various active sites (Table S11). These data show that the $\operatorname{HCP}(01 \overline{1} 1)$ and $\mathrm{HCP}(01 \overline{1} 2)$-oriented step-edge sites have activation barriers similar to the $\mathrm{HCP}(11 \overline{2} 1)$ surface, while the barriers for the terrace site with the FCC $(100)$ orientation and the step-edge sites with the FCC(211), FCC(110) and $\operatorname{HCP}(01 \overline{1} 3)$ orientations are slightly higher, and the barriers on the other surfaces are substantially higher. Other barriers involving hydrogenation and $\mathrm{CH}_{x}$ coupling of atomic carbon in the microkinetic model for the $\operatorname{HCP}(11 \overline{2} 1)$ model were scaled using the cobalt-carbon interaction energy as the main descriptor (Figure 4e). Herein, we used the same scaling approach as in our earlier works. ${ }^{32-34}$ The details of the implementation are shown in the Supporting Information. Sitebased reaction rates and surface coverages for each of the identified sites are given in Table $\mathrm{S} 12\left(523 \mathrm{~K}, \mathrm{H}_{2} / \mathrm{CO}\right.$ ratio 2$)$. As expected, a too strong cobalt-carbon interaction as found for the $\operatorname{FCC}(100), \operatorname{HCP}(01 \overline{1} 2)$, and $\operatorname{HCP}(01 \overline{1} 1)$ cobalt surfaces results in very low rates due to a high carbon coverage. Although the other candidate active sites also exhibit lower activities than the $\operatorname{HCP}(11 \overline{2} 1)$ model, the rates are within the range commonly reported for the FT reaction.

The estimated particle-based rates are presented as surface atom-based turnover frequencies (TOFs) in Figure 4f. The TOF is low for small particles and increases strongly with the size until a plateau is reached for particles above $5 \mathrm{~nm}$. This trend is consistent with many experimental observations made for the FT reaction on cobalt. It should be noted that the predicted rates are lower than observed in the experiment, which is most likely due to the accuracy limits of DFTcomputed energetics. By analyzing the contribution of each site to the TOFs (Figure S4, Supporting Information), we 


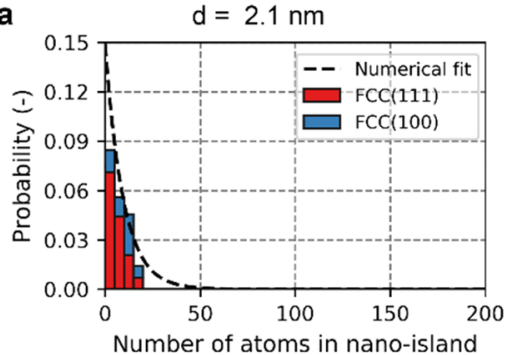

c
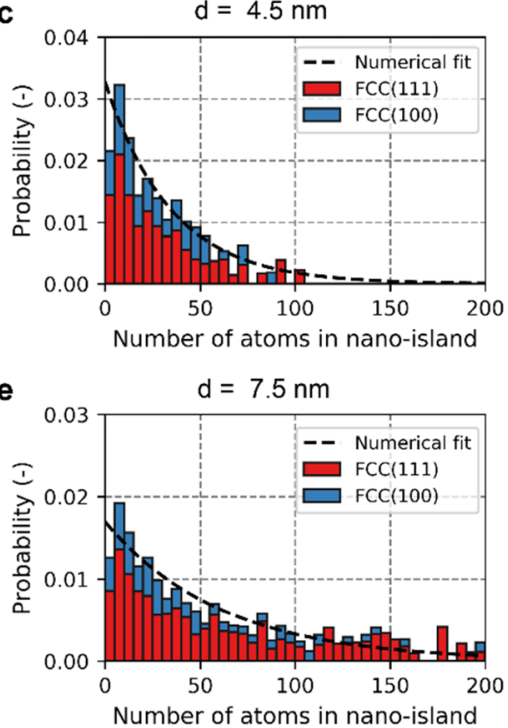

b

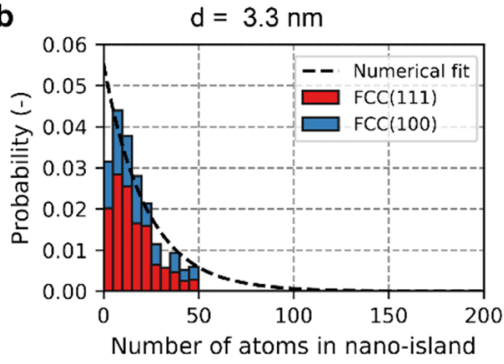

d

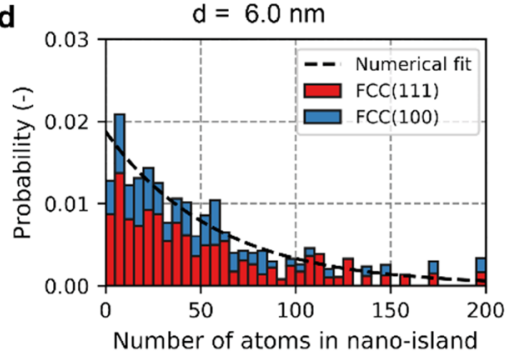

f

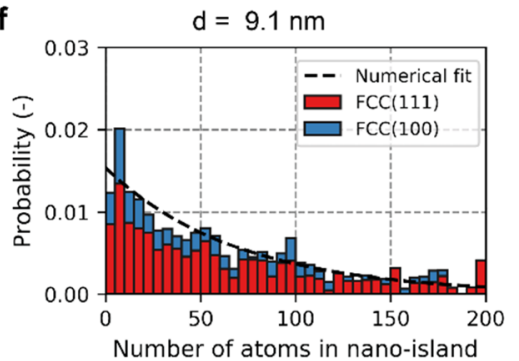

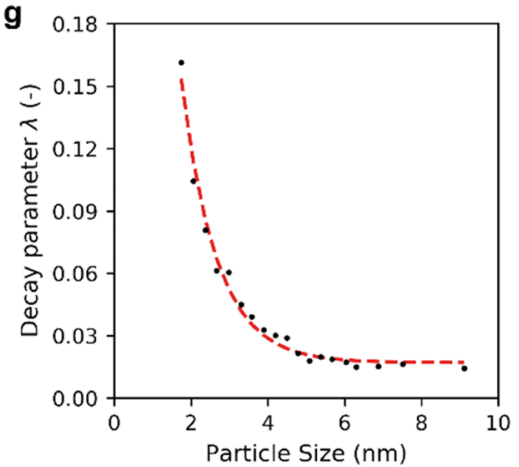

h

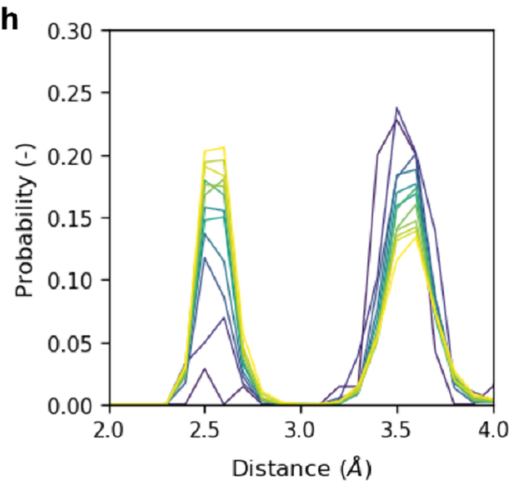

$\begin{aligned}-\mathrm{d} & =1.7 \mathrm{~nm}-\mathrm{d}=4.2 \mathrm{~nm}-\mathrm{d}=6.3 \mathrm{~nm} \\ \mathrm{~d} & =2.4 \mathrm{~nm}-\mathrm{d}=4.8 \mathrm{~nm}-\mathrm{d}=6.9 \mathrm{~nm} \\ \mathrm{~d} & =3.0 \mathrm{~nm}-\mathrm{d}=5.4 \mathrm{~nm}-\mathrm{d}=7.5 \mathrm{~nm} \\ \mathrm{~d} & =3.6 \mathrm{~nm}-\mathrm{d}=6.0 \mathrm{~nm}-\mathrm{d}=9.1 \mathrm{~nm}\end{aligned}$

Figure 6. Terrace nanoisland size probabilities for differently sized cobalt nanoparticles $(a-f)$, exponential decay parameter (lambda) as a function of the particle size $(\mathrm{g})$, and size-dependent FCC (111)-FCC (110) pair distribution function (h). (a) $2.1 \mathrm{~nm},(\mathrm{~b}) 3.3 \mathrm{~nm},(\mathrm{c}) 4.5 \mathrm{~nm},(\mathrm{~d}) 6.0 \mathrm{~nm}$, (e) $7.5 \mathrm{~nm}$, and (f) $9.1 \mathrm{~nm}$. FCC(111) and FCC(100)-oriented terrace nanoislands are shown in red and blue, respectively. The dotted lines are obtained by fitting an exponentially decaying function through the histograms. (g) Dotted red line serves as a guide to the eyes. (h) First peak (at $\sim 2.5 \AA$ ) of the pair distribution function indicates that these sites are direct neighbors.

conclude that $\mathrm{CO}$ conversion is mainly determined by stepedge sites with the FCC $(110)$ orientation.

Topological Analysis. Our findings clearly indicate that the unique particle size dependence observed for the FT reaction can be related to the increasing stability of a specific step-edge site on the surface of larger cobalt particles. Chorkendorff and co-workers suggested that extended facets on large metal particles can support terracelike overlayers, stabilizing step-edge sites at their interfaces. ${ }^{35}$ Figure 5 shows two-dimensional Robinson projections of the cobalt nanoparticles, emphasizing the FCC(110)-oriented step-edge sites and FCC(111) and FCC(100)-oriented terrace sites. These plots clearly demonstrate the increasing number of step-edge sites and their association with terrace overlayers for larger cobalt nanoparticles.

The occurrence of these overlayers as a function of their size (in terms of the number of surface cobalt atoms, $n$ ) is displayed in Figure $6 \mathrm{a}-\mathrm{f}$ for different cobalt nanoparticle sizes. Very small nanoparticles contain only very small ensembles of terraces. Larger cobalt nanoparticles stabilize larger overlayers. These data were further reduced into a single parameter $(\lambda)$ to describe the exponential decay of overlayers with respect to the size of the overlayers according to

$$
p=p_{0} \exp (-\lambda n)
$$

The decay parameter gives insight into the average size distribution of the nanoislands: a smaller value of the decay parameter implies that, on average, larger nanoislands are present. The size-dependent decay parameters are shown in Figure 6g. Herein, the absolute value of the decay parameter decreases with increasing particle size up to about $5 \mathrm{~nm}$ after which a plateau is observed.

To evaluate the hypothesis that the formation of nanoislands is linked to the formation of FCC(110)-oriented step-edge sites at the edges of these nanoislands, a pair distribution function was determined between the FCC(110)-oriented step-edge sites and the FCC(111)-oriented terrace sites, as shown in Figure $6 \mathrm{~h}$. The pair distance at $2.5 \AA$ reflects neighboring locations of the FCC(110) step-edge and the FCC(111) terrace sites, while those at larger distances reflect next-nearest neighbors, and so forth. Figure $6 \mathrm{~h}$ clearly shows that with increasing nanoparticle size, the first peak increases and the second peak decreases. Besides the larger terrace nanoislands, this result demonstrates that, on average, these nanoislands are positioned further apart with increasing particle size.

\section{CONCLUSIONS}

The present work demonstrates a general procedure for predicting structure sensitivity trends in heterogeneous catalysis by identifying catalytic ensembles at the surface of metal nanoparticles obtained by atomistic simulations. Simulated annealing is used, which is based on a force field trained by a large set of DFT data. Through pattern 
recognition, specific surface atom arrangements corresponding to reactive step-edge sites and unreactive terrace sites are identified and quantified as a function of the particle size. When applied to cobalt particles in the for catalysis interesting size range between 1 and $9 \mathrm{~nm}$, this approach shows that the density of FCC(110) step-edge sites increases strongly for particles from 1.4 to $6 \mathrm{~nm}$ and then levels off for larger particles. The surface-normalized FT activity of these cobalt particles displays qualitatively a similar trend to experimental data. A detailed analysis of the surface of these cobalt particles shows that the increasing number of proper step-edge sites for $\mathrm{CO}$ dissociation is associated with the formation of nanoislands with a terrace FCC(111) topology. These islands increase in size on larger particles up to $6 \mathrm{~nm}$. The constant density of step-edge sites on larger than $6 \mathrm{~nm}$ particles relates to the larger distance between the nanoislands.

\section{ASSOCIATED CONTENT}

\section{SI Supporting Information}

The Supporting Information is available free of charge at https://pubs.acs.org/doi/10.1021/acscatal.1c00651.

Cost function minimization data; DFT and force field energies; force field parameters; radial distribution functions; reference surface topologies; kinetic data; and activity contributions (PDF)

Example simulated annealing trajectory (MP4)

\section{AUTHOR INFORMATION}

\section{Corresponding Author}

Ivo A. W. Filot - Laboratory of Inorganic Materials and Catalysis, Department of Chemical Engineering and Chemistry, Eindhoven University of Technology, $5612 \mathrm{AZ}$ Eindhoven, the Netherlands; 10 orcid.org/0000-0003-14038379; Email: i.a.w.filot@tue.nl

\section{Authors}

Michel P. C. van Etten - Laboratory of Inorganic Materials and Catalysis, Department of Chemical Engineering and Chemistry, Eindhoven University of Technology, 5612 AZ Eindhoven, the Netherlands

Bart Zijlstra - Laboratory of Inorganic Materials and Catalysis, Department of Chemical Engineering and Chemistry, Eindhoven University of Technology, 5612 AZ Eindhoven, the Netherlands

Emiel J. M. Hensen - Laboratory of Inorganic Materials and Catalysis, Department of Chemical Engineering and Chemistry, Eindhoven University of Technology, $5612 \mathrm{AZ}$ Eindhoven, the Netherlands; (1) orcid.org/0000-0002-97542417

Complete contact information is available at:

https://pubs.acs.org/10.1021/acscatal.1c00651

\section{Author Contributions}

The manuscript was written through contributions of all authors. All authors have given approval to the final version of the manuscript.

\section{Notes}

The authors declare no competing financial interest.

\section{ACKNOWLEDGMENTS}

This work was supported by the Netherlands Center for Multiscale Catalytic Energy Conversion and NWO Gravitation program funded by the Ministry of Education, Culture and Science of the government of the Netherlands. The Netherlands Organization for Scientific Research is acknowledged for providing access to computational resources.

\section{ABBREVIATIONS USED}

CNA, common neighbor analysis; DFT, density functional theory; FCC, face centered cubic; FT, Fischer-Tropsch; HCP, hexagonal close-packed; $\mathrm{MD}$, molecular dynamics; $\mathrm{RF}_{3}$, reactive force field fitter; $\mathrm{RDF}$, radial distribution function; TEM, transmission electron microscopy; XRD, X-ray diffraction

\section{REFERENCES}

(1) Ligthart, D. A. J. M.; van Santen, R. A.; Hensen, E. J. M. Influence of particle size on the activity and stability in steam methane reforming of supported Rh nanoparticles. J. Catal. 2011, 280, 206220.

(2) Wei, J.; Iglesia, E. Structural requirements and reaction pathways in methane activation and chemical conversion catalyzed by rhodium. J. Catal. 2004, 225, 116-127.

(3) Parmaliana, A.; Arena, F.; Frusteri, F.; Coluccia, S.; Marchese, L.; Martra, G.; Chuvilin, A. L. Magnesia-Supported Nickel Catalysts. J. Catal. 1993, 141, 34-47.

(4) Spencer, N. D.; Schoonmaker, R. C.; Somorjai, G. A. Iron single crystals as ammonia synthesis catalysts: Effect of surface structure on catalyst activity. J. Catal. 1982, 74, 129-135.

(5) Honkala, K.; Hellman, A.; Remediakis, I. N.; Logadottir, A.; Carlsson, A.; Dahl, S.; Christensen, C. H.; Norskov, J. K. Ammonia Synthesis from First-Principles Calculations. Science 2005, 307, 555558.

(6) Karim, A. M.; Prasad, V.; Mpourmpakis, G.; Lonergan, W. W.; Frenkel, A. I.; Chen, J. G.; Vlachos, D. G. Correlating Particle Size and Shape of Supported $\mathrm{Ru} / \gamma-\mathrm{Al}_{2} \mathrm{O}_{3}$ Catalysts with $\mathrm{NH}_{3}$ Decomposition Activity. J. Am. Chem. Soc. 2009, 131, 12230-12239.

(7) Dahl, S.; Logadottir, A.; Egeberg, R.; Larsen, J. H.; Chorkendorff, I.; Tornqvist, E.; Norskov, J. Role of Steps in N2 Activation on $\mathrm{Ru}(0001)$. Phys. Rev. Lett. 1999, 83, 1814-1817.

(8) Bezemer, G. L.; Bitter, J. H.; Kuipers, H. P. C. E.; Oosterbeek, H.; Holewijn, J. E.; Xu, X.; Kapteijn, F.; van Dillen, A. J.; de Jong, K. P. Cobalt Particle Size Effects in the Fischer-Tropsch Reaction Studied with Carbon Nanofiber Supported Catalysts. J. Am. Chem. Soc. 2006, 128, 3956-3964.

(9) Kang, J.; Zhang, S.; Zhang, Q.; Wang, Y. Ruthenium Nanoparticles Supported on Carbon Nanotubes as Efficient Catalysts for Selective Conversion of Synthesis Gas to Diesel Fuel. Angew. Chem., Int. Ed. 2009, 48, 2565-2568.

(10) Carballo, J. M. G.; Yang, J.; Holmen, A.; Garcia-Rodriguez, S.; Rojas, S.; Ojeda, M.; Fierro, J. L. G. Catalytic effects of ruthenium particle size on the Fischer-Tropsch Synthesis. J. Catal. 2011, 284, 102-108.

(11) Den Breejen, J. P.; Radstake, P. B.; Bezemer, G. L.; Bitter, J. H.; Froseth, V.; Holmen, A.; de Jong, K. P. On the origin of the cobalt particle size effects in Fischer-Tropsch catalysis. J. Am. Chem. Soc. 2009, 131, 7197-7203.

(12) Iglesia, E.; Soled, S. L.; Fiato, R. A. Fischer-Tropsch synthesis on cobalt and ruthenium. Metal dispersion and support effects on reaction rate and selectivity. J. Catal. 1992, 137, 212-224.

(13) Tuxen, A.; Carenco, S.; Chintapalli, M.; Chuang, C.; Escudero, C.; Pach, E.; Jiang, P.; Borondics, F.; Beberwyck, B.; Alivisatos, A. P.; Thornton, G.; Pong, W.; Guo, J.; Perez, R.; Besenbacher, F.; Salmeron, M. Size-dependent dissociation of carbon monoxide on cobalt nanoparticles. J. Am. Chem. Soc. 2013, 135, 2273-2278.

(14) van Santen, R. A.; Neurock, M.; Shetty, S. G. Reactivity theory of transition-metal surfaces: A brønsted-evans- polanyi linear activation energy-free-energy analysis. Chem. Rev. 2010, 110, 20052048. 
(15) Sun, G.; Sautet, P. Metastable Structures in Cluster Catalysis from First-Principles: Structural Ensemble in Reaction Conditions and Metastability Triggered Reactivity. J. Am. Chem. Soc. 2018, 140, 2812-2820.

(16) Cheula, R.; Maestri, M.; Mpourmpakis, G. Modeling Morphology and Catalytic Activity of Nanoparticle Ensembles Under Reaction Conditions. ACS Catal. 2020, 10, 6149-6158.

(17) Zhao, P.; Cao, Z.; Liu, X.; Ren, P.; Cao, D.; Xiang, H.; Jiao, H.; Yang, Y.; Li, Y.; Wen, X. Morphology and Reactivity Evolution of $\mathrm{HCP}$ and FCC Ru Nanoparticles under CO Atmosphere. ACS Catal. 2019, 9, 2768-2776.

(18) Van Helden, P.; Ciobîce, I. M.; Coetzer, R. L. J. The sizedependent site composition of FCC cobalt nanocrystals. Catal. Today 2016, 261, 48-59.

(19) Agrawal, R.; Phatak, P.; Spanu, L. Effect of phase and size on surface sites in cobalt nanoparticles. Catal. Today 2018, 312, 174180.

(20) Rahm, J. M.; Erhart, P. Beyond Magic Numbers: Atomic Scale Equilibrium Nanoparticle Shapes for Any Size. Nano Lett. 2017, 17, 5775-5781.

(21) Chuela, R.; Soon, A.; Maestri, M. Prediction of morphological changes of catalyst materials under reaction conditions by combined $\mathrm{ab}$ initio thermodynamics and microkinetic modelling. Catal. Sci. Technol. 2018, 8, 3493-3503.

(22) Matveev, V. V.; Baranov, D. A.; Yurkov, G. Y.; Akatiev, N. G.; Dotsenko, I. P.; Gubin, S. P. Cobalt nanoparticles with preferential hcp structure: A confirmation by X-ray diffraction and NMR. Chem. Phys. Lett. 2006, 422, 402-405.

(23) Van Duin, A. C. T.; Dasgupta, S.; Lorant, F.; Goddard, W. A. ReaxFF: A Reactive Force Field for Hydrocarbons. J. Phys. Chem. A 2001, 105, 9396-9409.

(24) Chenoweth, K.; van Duin, A. C. T.; Goddard, W. A. ReaxFF Reactive Force Field for Molecular Dynamics Simulations of Hydrocarbon Oxidation. J. Phys. Chem. A 2008, 112, 1040-1053.

(25) Mills, G.; Jónsson, H.; Schenter, G. K. Reversible work transition state theory: application to dissociative adsorption of hydrogen. Surf. Sci. 1995, 324, 305-337.

(26) Henkelman, G.; Uberuaga, B. P.; Jónsson, H. A climbing image nudge elestic band method for finding saddle points and minimum energy paths. J. Chem. Phys. 2000, 113, 9901-9904.

(27) Henkelman, G.; Jónsson, H. Improved tangent estimate in the nudged elastic band method for finding minimum energy paths and saddle points. J. Chem. Phys. 2000, 113, 9978-9985.

(28) Matsumoto, M.; Nishimura, T. Mersenne Twister: A 623Dimensionally Equidistributed Uniform Pseudo-Random Number Generator. ACM Trans. Model. Comput. Simul. 1998, 8, 3-30.

(29) Reinhart, W. F.; Long, A. W.; Howard, M. P.; Ferguson, A. L.; Panagiotopoulos, A. Z. Machine learning for autono-mous crystal structure identification. Soft Matter 2017, 13, 4733-4745.

(30) Karaca, H.; Hong, J.; Fongarland, P.; Roussel, P.; GribovalConstant, A.; Lacroix, M.; Hortmann, K.; Safonova, O. V.; Khodakov, A. Y. In situ XRD investigation of the evolution of alumina-supported cobalt catalysts under realistic conditions of Fischer-Tropsch synthesis. Chem. Commun. 2010, 46, 788-790.

(31) Kitakami, O.; Sato, H.; Shimada, Y.; Sato, F.; Tanaka, M. Size effect on the crystal phase of cobalt fine particles. Phys. Rev. B 1997, 56, 13849-13854.

(32) Zijlstra, B.; Broos, R. J. P.; Chen, W.; Bezemer, G. L.; Filot, I. A. W.; Hensen, E. J. M. The vital role of step-edge sites for both CO activation and chain growth on cobalt Fischer-Tropsch catalysts revealed through first-principles based microkinetic modeling including lateral interactions. ACS Catal. 2020, 10, 9376-9400.

(33) Zijlstra, B.; Broos, R. J. P.; Chen, W.; Oosterbeek, H.; Filot, I. A. W.; Hensen, E. J. M. Coverage effects in $\mathrm{CO}$ dissociation on Metallic Cobalt Nanoparticles. ACS Catal. 2019, 9, 7365-7372.

(34) Filot, I. A. W.; van Santen, R. A.; Hensen, E. J. M. The optimally performing Fischer-Tropsch catalyst. Angew. Chem. 2014, 126, 12960-12964.
(35) Strebel, C.; Murphy, S.; Nielsen, R. M.; Nielsen, J. H.; Chorkendorff, I. Probing the active sites for CO dissociation on ruthenium nanoparticles. Phys. Chem. Chem. Phys. 2012, 14, 80058012. 